\title{
Ambulatory blood pressure monitoring
}

\author{
W R Nicholson, J N S Matthews, J J O’Sullivan, C Wren
}

\begin{abstract}
Ambulatory blood pressure monitoring (ABPM) in adults is proving to be useful. The aim of this study was to determine if ABPM is accurate in the lower blood pressure range encountered in children and, equally important, whether it is acceptable to children. Thirty one children, between the ages of 6 and 18 years, were assessed using an ambulatory blood pressure monitor that uses an auscultatory method. Blood pressure was measured in the contralateral arm with a mercury sphygmomanometer and an oscillometric device at the beginning and end of the study for comparison. Over a blood pressure range of $90-130 \mathrm{~mm} \mathrm{Hg}$ systolic and $40-80 \mathrm{~mm} \mathrm{Hg}$ diastolic, a close agreement was found with the sphygmomanometer; the limits of agreement ( \pm 2 SD) were $11.6 \mathrm{~mm} \mathrm{Hg}$ for systolic blood pressure and $13.6 \mathrm{~mm} \mathrm{Hg}$ for diastolic blood pressure. The bias was less than $1.0 \mathrm{~mm} \mathrm{Hg}$. The ambulatory device was worn by all patients for at least 16 hours with an average of 52 recordings per patient. The majority found the device comfortable to wear and were not woken from sleep.

(Arch Dis Child 1993; 69: 681-684)
\end{abstract}

The regular measurement of blood pressure is just as important in childhood as in adult life. However, there are a number of problems that are particularly important in childhood. Firstly, blood pressure varies with age and although population norms have been published, ${ }^{1-3}$ there is considerable variation and consequently no internationally agreed standards. ${ }^{4}$ Secondly, the size of the cuff must be carefully matched to the size of the arm. It is recommended that the bladder width should be 40 to $50 \%$ of mid-arm circumference, ${ }^{5}$ although in clinical practice such precision is rarely achieved. Finally, the fourth and fifth Korotkoff sounds are frequently found to occur simultaneously and the fifth phase may not occur at all, adding further imprecision to the measurement of diastolic blood pressure in children.

Hypertension in children can be defined as the state in which the average systolic and/or average diastolic blood pressure is greater than the 95th centile for age and sex with measurements obtained on at least three occasions. ${ }^{6}$ The prevalence of hypertension in children is of the order of 1 to $3 \%$. Of these children one tenth will have severe hypertension and in the majority the increased blood pressure will be secondary to renal disease or coarctation of the aorta. $^{7}$
Currently the detection of hypertension is based on periodic blood pressure measurements or the blood pressure response to exercise testing. Continuous recording of interarterial blood pressure has shown little relationship to sporadic measurements and arterial recordings are obviously not appropriate or available for routine clinical use in children. Recent technological advances have produced non-invasive ambulatory blood pressure recorders capable of multiple sampling of blood pressure via sphygmomanometer cuff with the storage of data for later analysis. These devices have been validated in adults and there is now a considerable body of literature reporting their use. ${ }^{89}$

The aims of our study were to determine whether ambulatory blood pressure monitoring (ABPM) is accurate in the lower blood pressure range encountered in childhood and, equally important, whether it was acceptable to children.

\section{Patients and methods}

\section{PATIENTS}

Our study population consisted of 31 children aged 6 to 18 years with a median age of 11 years. There were 15 females. Twenty six were inpatients and the remainder outpatients. None of these children was obese (greater than or equal to $120 \%$ ideal body weight based on height age). All but five were normotensive (blood pressure less than 90th centile). One child was on antihypertensive medication. Four patients had successfully undergone cardiac transplantation. The majority were electively admitted for cardiac catheterisation or surgery.

\section{EQUIPMENT}

We assessed the A\&D ambulatory blood pressure monitor TM $2420 / T M 2020$, which is a portable non-invasive recorder $(4 \times 6.5 \times 14 \mathrm{~cm}$ in size, $390 \mathrm{~g}$ in weight) powered by a rechargeable battery. There are two cuff sizes, child (bladder width $9.0 \mathrm{~cm}$ and length 16.0 $\mathrm{cm}$ ) or adult (bladder width $12.0 \mathrm{~cm}$ and length $22.0 \mathrm{~cm}$ ) The cuff is pressurised by a miniature, low noise rotary micropump. To eliminate noises resulting from body motion, two microphones are used to distinguish Korotkoff sounds. The first and fifth Korotkoff sounds are taken as systolic and diastolic pressures respectively. Systolic blood pressure, diastolic blood pressure, and heart rate are measured automatically at intervals of $1-60$ minutes throughout 24 hours. These data can be stored as many as 600 times in the recorder's semiconductor memory. After measurement, mean (SD) values and trend 


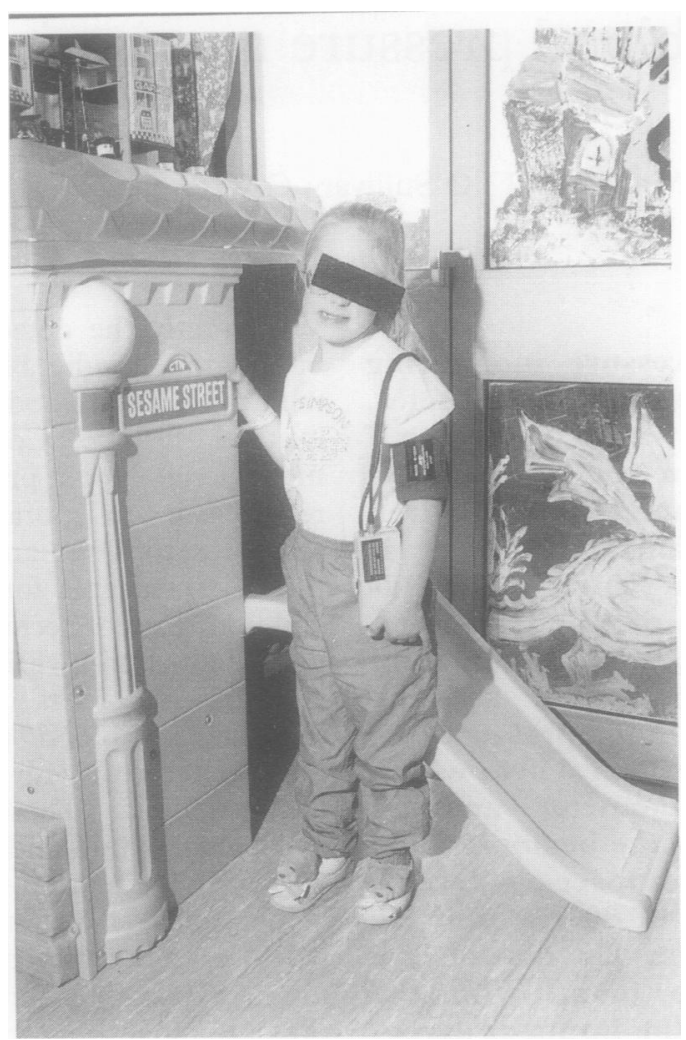

Figure 1 A 5 year old girl wearing the recorder. This is a lightweight device and can easily be concealed under clothing if desired.

graphs of systolic blood pressure, diastolic blood pressure, and heart rate are printed out by means of a miniature analyser measuring $5 \times 7.5 \times 15 \mathrm{~cm}$. Error readings are indicated by one of 12 codes. The machines measure in a range for systolic pressure of 60 to $280 \mathrm{~mm} \mathrm{Hg}$ with a diastolic pressure of 40 to $160 \mathrm{~mm} \mathrm{Hg}$. Readings are edited if they fall outside this range or if the pulse pressure is less than 10 or greater than $150 \mathrm{~mm} \mathrm{Hg}$. This is the quietist (noise $40 \mathrm{~dB}$ ) and the lightest product of its kind on the market currently. Figure 1 illustrates a 5 year old girl wearing the recorder and the analyser coupled to the recorder reading to program or retrieve data.

\section{PROTOCOL}

This project was approved by the joint ethics committee of the Newcastle Health Authority. After informed consent was obtained the patient was fitted with the recorder and the cuff attached to the non-dominant arm. Selection of cuff size was based on measurement of the mid-arm circumference and corresponded to the manufacturer's recommendation. At the beginning and/or end of the 24 hour period comparison measurements were made in the contralateral arm using the same cuff size and measured by both oscillometric method (Dinamap) and a standard mercury sphygmomanometer. Each instrument was used in random order. Three measurements were taken with each device over a period of five minutes and averaged for statistical comparison. Automatic recordings were made every 30 minutes during the day time and every 60 minutes at night. The child
Comparisons of ABPM with measurement by standard mercury sphygmomanometer and an oscillometric device (Dinamap); limits of agreement are mean $\pm 2 S D$

\begin{tabular}{|c|c|c|c|c|}
\hline & \multicolumn{2}{|c|}{ Sphygomanometer } & \multicolumn{2}{|c|}{ Dinamap } \\
\hline & Systolic & Diastolic & Systolic & Diastolic \\
\hline $\begin{array}{c}\text { Differenc } \\
\text { Mean } \\
\text { SD }\end{array}$ & $\begin{array}{c}\text { lood pres } \\
0.56 \\
5 \cdot 8\end{array}$ & $\begin{array}{c}\mathrm{re}\left(\mathrm{mm} \mathrm{H} \mathrm{H}_{\mathrm{g}}\right. \\
0.80 \\
6.8\end{array}$ & $\begin{array}{c}-6 \cdot 10 \\
7 \cdot 2\end{array}$ & $\begin{array}{l}1.00 \\
8.5\end{array}$ \\
\hline $\begin{array}{c}\% \text { Readir } \\
<5 \\
<10 \\
<15\end{array}$ & $\begin{array}{c}\text { ffering by } \\
56 \\
87 \\
100\end{array}$ & $\begin{array}{l}\mathrm{nm} \mathrm{Hg}) \\
54 \\
85 \\
95\end{array}$ & $\begin{array}{l}46 \\
73 \\
88\end{array}$ & $\begin{array}{l}44 \\
73 \\
90\end{array}$ \\
\hline
\end{tabular}

was instructed to remain still with the arm extended during the recording. The time each child fell asleep and awoke was recorded by a nurse or parent to the nearest 15 minutes. Finally, each child was asked what they thought of the device and whether it awoke them from sleep.

\section{Results}

ACCURACY

Comparisons between the methods was made using the technique of Bland and Altman. ${ }^{10}$ These data are summarised in the table. Requirements for validation of the accuracy of ABPM have been proposed. ${ }^{11}$ It is suggested that the mean difference of the paired measurements by the monitor and reference technique should be within $\pm 5 \mathrm{~mm} \mathrm{Hg}$ with a standard deviation of the difference between techniques to be $\leqslant 8 \mathrm{~mm} \mathrm{Hg}$. It can be seen that using these criteria there is a satisfactory agreement between ABPM and measurement by the standard mercury sphygmomanometer but not with the Dinamap recorder.

\section{ERRORS}

Error readings occurred in $16 \%$ of all recordings (range $0-46 \%$ ). Six whole day recordings were rejected because they had more than $25 \%$ errors. The patient who had an unusually high number of error recordings (20/57) possibly had a recorder malfunction. The machine continued to register a wide pulse pressure without interruption for a period of four hours. The commonest reason for an error reading was failure of the microphone to detect the Korotkoff sounds $(42 \%)$ followed by a wide pulse pressure $(21 \%)$.

\section{ACCEPTABILITY}

Thirty six children were initially approached to wear the device (fig 2); in two it failed to record. A further three disliked the machine after an initial trial. We therefore had 31 patients who completed a whole day record of at least 16 hours with a mean duration of 22.5 hours. One child, the youngest in the study, asked to have the machine taken off. In all other situations where recordings terminated short of 24 hours this was due to ward routine, for example, a child going to theatre or being discharged. The average number of blood pressure recordings made by the monitor was 52 per patient. Six children were woken briefly 


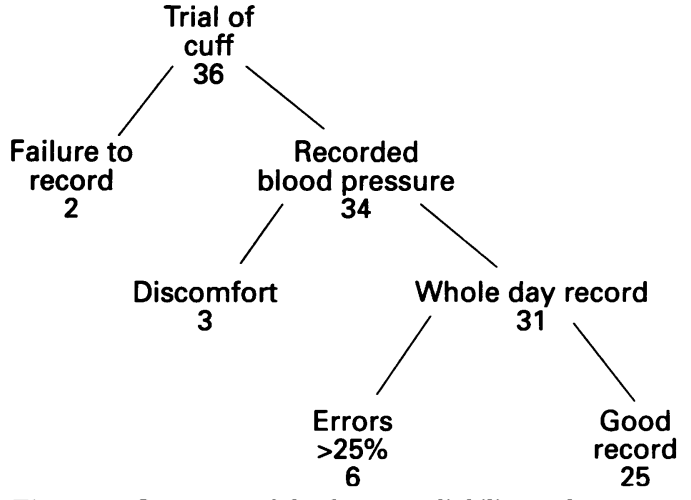

Figure 2 Summary of the data on reliability and acceptability for whole day recordings (greater than 16 hours) with $A B P M$.

from sleep but only two because of discomfort. Twenty five children said they would wear the device again.

\section{WHOLE DAY RECORD}

Figure 3 shows a typical profile of a whole day ambulatory blood pressure record. The patient was an 11 year old boy who had successfully undergone cardiac transplantation three years previously. He was normotensive with a blood pressure of $110 / 65 \mathrm{~mm} \mathrm{Hg}$ in clinic. He was an outpatient during this study. Note the variability of his systolic blood pressure rising above $200 \mathrm{~mm} \mathrm{Hg}$ at 1800 hours when he was at a disco and at 0900 hours when he went to school.

\section{Discussion}

The A\&D ambulatory blood pressure monitor has previously been validated in adults by Tochikubo et al. ${ }^{12}$ Sixty two normal adults between the age of 25 and 60 years were assessed. Simultaneous measurements with a mercury sphygmomanometer and the monitor were compared. Over a blood pressure range of 75-200 mm $\mathrm{Hg}$ systolic blood pressure and $50-125 \mathrm{~mm} \mathrm{Hg}$ diastolic blood pressure very close agreement was found, the limits of

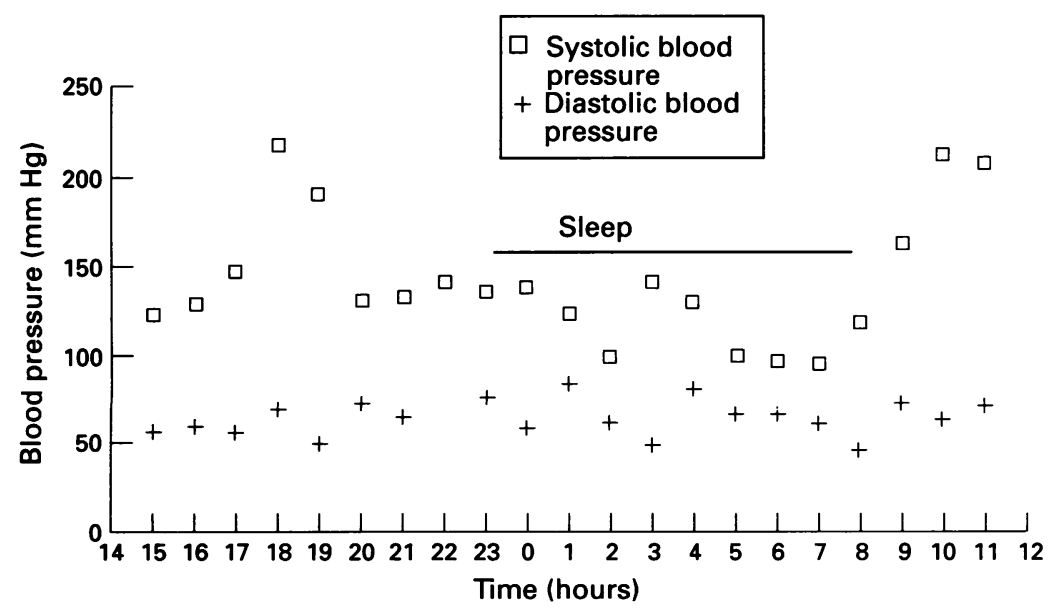

Figure 3 Whole day record of an 11 year old boy. His mean blood pressure in outpatient clinic was $110 / 65 \mathrm{~mm} \mathrm{Hg}$. Note the variability in systolic blood pressure during a normal day. Between 1730 and 1900 he was at a disco but did not dance. He awoke briefly at 0300; note the early morning rise in blood pressure before awakening. He was driven to school in the morning and started class at 0900. agreement being $\pm 9.4 \mathrm{~mm} \mathrm{Hg}$ for systolic blood pressure and $\pm 10.0 \mathrm{~mm} \mathrm{Hg}$ for diastolic blood pressure, the bias being 1.2 and $2.7 \mathrm{~mm}$ $\mathrm{Hg}$ respectively.

There are few studies of ambulatory blood pressure monitoring in children. Daniels et al assessed the Del Mar Avionics Ambulatory Pressurometer III on a group of 84 hypertensive children aged 6 to 23 years (mean age $14 \cdot 8$ years). ${ }^{13}$ This device also ausultates the Korotkoff signs but with a single microphone. They compared readings at the beginning and end of the 24 hour period with sphygmomanometer readings taken simultaneously via a connecting jack. After the initial readings they calibrated the device to read within $5 \mathrm{~mm}$ of the sphygmomanometer reading. Despite this, the median difference at the beginning of the assessment was $5.3 \mathrm{~mm} \mathrm{Hg}$ for systolic blood pressure (range 1.0-21.4 $\mathrm{mm} \mathrm{Hg}$ ) and $4.7 \mathrm{~mm} \mathrm{Hg}$ for diastolic blood pressure (range $1 \cdot 2-11.5 \mathrm{~mm} \mathrm{Hg}$ ). Recordings were made every 7.5 minutes and each child had on average 178 measurements. Interestingly, this machine weighed over $2 \mathrm{~kg}$ and no mention of patient acceptability was made.

Portman et al report their use of an oscillometric ABPM device (Space Labs 90202) in a group of 99 normal 10 year olds. ${ }^{14}$ Diaries enabled retrospective assessment of activity level and emotional state. This device had a similar accuracy to ours but they found systolic blood pressure measured by their device was $5 \mathrm{~mm} \mathrm{Hg}$ higher than comparison measurements with a mercury manometer. It is now clear that oscillometric ABPM devices record a wider pulse pressure than auscultatory monitors, ${ }^{15}$ and their validation may have improved by comparison with a standard oscillometric blood pressure recorder such as Dinamap. Their device was also lightweight and quiet but $27 \%$ reported sleep disturbance and their error rate was high at $27 \%$. This type of ABPM appears to be limited by vibratory interference in active children. ${ }^{16}$

Loirat et al used a Nippon Colin monitor 630 that simultaneously obtains blood pressure values by the auscultatory and oscillometric methods. ${ }^{15}$ This reduced their error rate to a very acceptable $2 \cdot 7 \%$ for both methods combined. This group went on to assess 28 children, aged 8-17 years, with borderline hypertension. For reasons we discuss later, interpretation of data from this type or application is fraught with difficulty as it is not clear what is an abnormal or 'hypertensive' ABPM profile.

Our study does show a reasonable agreement between a state of the art ambulatory blood pressure monitor and two generally accepted non-invasive techniques. It complies with the criteria of O'Brien et al ${ }^{11}$ and compares favourably with two other devices used in children. ${ }^{1415}$ 'White coat' hypertension exists in children just as it does in adults. ${ }^{17} 18$ It is therefore likely that this new technology will prove useful in the assessment and management of hypertension in children and adolescence. However, a number of important 
questions need to be answered before ABPM is recommended for routine clinical use in childhood. (1) Is there now an established need for this new methodology? Are the current techniques of blood pressure measurement inadequate? (2) Is the equipment reliable and practical enough to give useful data? (3) Is it safe for and acceptable to children? (4) Is there an adequate definition of a normal or an abnormal result? Do we have the basis for diagnostic interpretation of results for an individual patient? (5) Is it cost effective? Do the benefits of the technique outweigh the expense? We have attempted to address the first three questions in our present study.

An enormous amount of data is generated by this technique and it is not clear how this should be handled and interpreted. A mean 24 hour systolic and diastolic blood pressure is commonly quoted in adult studies but it seems to be an over simplification that conceals important information about variability. An alternative might be to record mean sleeping and awake blood pressures or present a summated profile with hourly or two hourly average blood pressure plotted against time (fig 3).

In conclusion ABPM in children gives results that are reliable and most children over the age of 6 years find this investigation acceptable. Further research is needed to establish how to make the most effective use of this new technology.
1 Andre JL, Deschamps JP, Gueguen R. La tension arterielle chez l'infant et l'adolescent. Arch Fr Pediatr 1980; 37: 477-82.

2 Voors AW, Foster TA, Frericks RR, et al. Studies of blood pressure in children age 5-14 years. The Bogalusa heart study. Circulation 1976; 54: 319-27.

3 Cassimos C, Varlamis G, Karamperis S, et al. Blood pressure in children and adolescents. Acta Paediatr Scand 1977; 66: 403-4.

4 Dillon MJ. Blood pressure. Arch Dis Child 1988; 63: 347-9. 5 Park MK Paediatric cardiology for practitioners. Chicago: Yearbook Medical Publishers, 1988: 13-5.

6 Report of the second task force on blood pressure control in children. Pediatrics 1987; 79: 1-25.

7 Dillon MJ. Investigation and management of hypertension in children. Pediatr Nephrol 1987; 1: 59-68.

8 National High Blood Pressure Education Program Coordinating Committee. National high blood pressure education program working group report on ambulatory blood pressure monitorings. Arch Intern Med 1990; 150: 2270-80.

9 Weber MA, ed. Clinical application of automated wholeday blood pressure monitoring. (Proceedings of a day blood pressure monitoring. (Proceeding

10 Bland JM, Altman DG. Statistical methods for assessing agreement between two methods of clinical measurement. Lancet 1986; i: 307-10

11 O'Brien E, O'Malley K, Sheridan J. The need for a standardised protocol for validating non invasive ambulatory blood pressure measuring devices. $\mathcal{f}$ Hypertens 1989 ; 7 (suppl): 19-20.

12 Tochikubo O, Minamisawa $\mathrm{K}$, Miyajima E, et al. A new compact 24 hour indirect blood pressure recorder and its clinical application. Ipn Heart f 1988; 29: 257-69.

13 Daniels SR, Loggie JM, Burton T, et al. Difficulties with ambulatory blood pressure monitoring in children and ambulatory blood pressure monitoring in

14 Portman RJ, Yetman RJ, West MS. Efficacy of 24-hour ambulatory blood pressure monitoring in children. F Pediatr 1991; 118: 842-9.

15 Loirat C, Azancot-Benisty A, Bossu C. Value of ambulatory blood pressure monitoring in borderline hypertension in the child. Ann Pediatr (Paris) 1991; 38: 381-6.

16 Jacoby AC, Fixler DE, Torres EJ. Limitations of an oscillometric ambulatory blood pressure monitor in physically active children. $\mathcal{F}$ Pediatr 1993; 122: 2:231-6.

17 Hornsby JL, Mongan PF, Taylor AT, et al. 'White coat' hypertension in children. $\mathcal{F}$ Fam Pract 1991; 33: 617-23.

18 Waeber B, Niederberger M, Nussberger J, et al. Ambulatory blood pressure monitoring in children, adolescents and elderly people. f Hypertens Suppl 1991; 9: 872-4. 